This is an accepted manuscript of an article published by Taylor \& Francis in International Journal of General Systems on December 8 2017, available online: https://doi.org/10.1080/03081079.2017.1410147

\title{
ARTICLE \\ Multivalued contexts associated with criteria
}

\author{
C. Alcalde ${ }^{a *}$, A. Burusco ${ }^{b, c}$ \\ ${ }^{a}$ Dept. Matemática Aplicada. Universidad del País Vasco UPV/EHU. Spain; \\ ${ }^{b}$ Dept. Automática y Computación, Univ. Pública de Navarra. Spain; \\ ${ }^{c}$ Institute of Smart Cities, Universidad Pública de Navarra. Spain
}

(July 5, 2017)

\begin{abstract}
We are interested in the study of $L$-fuzzy contexts taking into account different criteria. These contexts arise when we want to analyze the relationship between objects and attributes from different points of view. Furthermore, in some occasions these $L$-fuzzy contexts have several values for every pair object-attribute. We will see how both the WOWA operators and the Choquet integrals will be interesting tools for the aggregation processes that we are going to carry out.
\end{abstract}

Keywords: $L$-fuzzy concept analysis, $L$-fuzzy concepts associated with criteria, WOWA operators, Choquet integral.

\section{Introduction}

The Formal Concept Analysis (Wille 1982) extracts information from a binary table that represents a formal context $(X, Y, R)$ with $X$ and $Y$ finite sets of objects and attributes respectively and $R \subseteq X \times Y$. The hidden information consists of pairs $(A, B)$ with $A \subseteq X$ and $B \subseteq Y$, called formal concepts, verifying $A^{*}=B$ and $B^{*}=A$, where $(\cdot)^{*}$ is the derivation operator that associates the attributes related to the elements of $A$ to every object set $A$, and the objects related to the attributes of $B$ to every attribute set $B$. These formal concepts can be interpreted as a group of objects $A$ that shares the attributes of $B$.

In previous works (Burusco and Fuentes-González 1994, 1998) we have defined the $L$-fuzzy contexts $(L, X, Y, R)$, with $L$ a complete lattice, $X$ and $Y$ sets of objects and attributes respectively and $R \in L^{X \times Y}$ a fuzzy relation between the objects and the attributes. This is an extension of the formal contexts of Wille when we want to study the relations between the objects and the attributes with values in a complete lattice $L$, instead of binary ones.

The $L$-fuzzy concept analysis (Burusco and Fuentes-González 1994, 1998) provides a tool for the extraction of knowledge from data tables ( $L$-fuzzy contexts) using $L$ fuzzy concepts.

This theory can be considered as a tool of multicriteria decision making (Fodor and Roubens 1994). The classical multicriteria decision making takes a set of alternatives and a set of points of view (called criteria) each of them having a weight.

\footnotetext{
${ }^{*}$ Corresponding author. Email: c.alcalde@ehu.es
} 
The procedures perform generally in two steps: aggregation and exploitation. The aggregation phase defines an outranking relation which indicates the global preference between every ordered pair of alternatives taking into consideration the weights of the different points of view. The exploitation phase transforms the global information about alternatives into a global ranking of them using different ways.

The difference between the classical multicriteria decision making and our theory is that we have three different sets: objects, attributes and criteria, and two of them are related by means of a relation $R$ that is the $L$-fuzzy context. However, we can also study the possibility of setting up a ranking in the object or in the attribute sets as it is done in multicriteria decision making.

We are also interested in the study of contexts with several observations. In the past (Burusco and Fuentes-González 1997) the $L$-fuzzy contexts obtained from several expert opinions were analyzed. In that case multisets were used as a tool for working in that situation.

Furthermore, the election of a good aggregation operator can be crucial to obtain a more complete information from the $L$-fuzzy contexts in some specific situations. In the paper, we will see that some results obtained in the $L$-fuzzy concept analysis can be improved using WOWA operators and Choquet integrals. The first one allows the use of two weighting vectors, one for the observations and the other for the experts. With the last one, we can give a weight not only for particular observations but also for groups.

Summarizing, the study of the $L$-fuzzy contexts associated with criteria and with the possibility of having several observations for each object-attribute pair is the main objective of this work. In order to do this, we begin recovering the most important results about $L$-fuzzy concept analysis, WOWA operators and Choquet integrals that will be useful in this work.

\subsection{L-fuzzy concept analysis}

In our papers (Burusco and Fuentes-González 1994, 1998), we have defined the derivation operators 1 and 2 given by means of these expressions:

$\forall A \in L^{X}, \forall B \in L^{Y}$

$$
\begin{aligned}
& A_{1}(y)=\inf _{x \in X}\{I(A(x), R(x, y))\} \\
& B_{2}(x)=\inf _{y \in Y}\{I(B(y), R(x, y))\}
\end{aligned}
$$

being $I$ a fuzzy implication operator defined in the lattice $(L, \leq)$.

The information stored in the $L$-fuzzy context is visualized by means of the $L$ fuzzy concepts which are pairs $\left(A, A_{1}\right) \in L^{X} \times L^{Y}$ with $A \in f i x(\varphi)$, set of fixed points of the operator $\varphi$, being defined from the derivation operators 1 and 2 as $\varphi(A)=\left(A_{1}\right)_{2}=A_{12}$. These pairs, whose first and second components are said to be the fuzzy extension and intension respectively, represent a group of objects that share a group of attributes.

Using the usual order relation between fuzzy sets, that is,

$$
\forall A, C \in L^{X}, \quad A \leq C \Longleftrightarrow A(x) \leq C(x) \quad \forall x \in X,
$$


we define the set $\mathcal{L}=\left\{\left(A, A_{1}\right) / A \in f i x(\varphi)\right\}$ with the order relation $\preceq$ : $\forall\left(A, A_{1}\right),\left(C, C_{1}\right) \in \mathcal{L},\left(A, A_{1}\right) \preceq\left(C, C_{1}\right)$ if $A \leq C\left(\right.$ or $\left.C_{1} \leq A_{1}\right)$.

We proved that $(\mathcal{L}, \preceq)$ is a complete lattice that is said to be the $L$-fuzzy concept lattice (Burusco and Fuentes-González 1994, 1998).

On the other hand, given $A \in L^{X}$, (or $B \in L^{Y}$ ) we can obtain the associated $L$-fuzzy concept applying twice the derivation operators. In the case of using a residuated implication, the associated $L$-fuzzy concept is $\left(A_{12}, A_{1}\right)$ (or $\left(B_{2}, B_{21}\right)$ ).

Other important papers generalize the Formal Concepts Analysis using residuated implication operators (Bělohlávek 1999; Bělohlávek, Vychodil 2005; Polland 1997). Moreover, there are extensions of Formal Concept Analysis to the interval-valued case (Burusco and Fuentes-González 2001; Alcalde et al. 2009; Djouadi and Prade 2010, 2011) and to the fuzzy property-oriented and multi-adjoint concept lattices framework (Medina and Ojeda-Aciego 2010; Medina 2012; Medina and Ojeda-Aciego 2013). Finally, we can also find studies of the evolution in time of $L$-fuzzy contexts (Alcalde et al. 2016).

Next, we introduce the WOWA operators.

\subsection{WOWA operators}

Firstly we show the definitions of the OWA operators:

Definition 1. (Yager 1988) A mapping $F_{w}$ from $L^{n} \longrightarrow L$, where $L=[0,1]$ is called an OWA operator of dimension $n$ if associated with $F_{w}$ is a weighting $n$-tuple $w=\left(w_{1}, w_{2}, \ldots, w_{n}\right)$ such that $w_{i} \in[0,1]$ and $\sum_{1 \leq i \leq n} w_{i}=1$, where $F_{w}\left(a_{1}, a_{2}, \ldots, a_{n}\right)=w_{1} \cdot b_{1}+w_{2} \cdot b_{2}+\cdots+w_{n} . b_{n}$, with $b_{i}$ the ith largest element in the collection $a_{1}, a_{2}, \ldots, a_{n}$.

We applied these OWA operators to the fuzzy contexts sequences to analyze tendencies when the sequence represents the evolution in time.

After that, the Weighted OWA operators (WOWA) were defined (Torra 1997) and combine the advantages of the OWA operators and the ones of the weighted mean (Calvo and Mesiar 2003a,b). These operators consider two weighting vectors: $w=\left(w_{1}, w_{2}, \ldots, w_{n}\right)$ corresponding to the relevance of the values (operator OWA) and $p=\left(p_{1}, p_{2}, \ldots, p_{n}\right)$ corresponding to the relevance of the sources or experts.

As particular cases, if $w_{i}=1 / n, i \in\{1, \ldots, n\}$ then we have the weighted mean with $p$ and if $p_{i}=1 / n, i \in\{1, \ldots, n\}$, an OWA operator with $w$.

This is the definition:

Definition 2. (Torra 1997) Let $p, w$ be weighting vectors of dimension $n, p=$ $\left(p_{1}, p_{2}, \ldots, p_{n}\right)$ and $w=\left(w_{1}, w_{2}, \ldots, w_{n}\right)$ such that $p_{i}, w_{i} \in[0,1]$ and $\sum_{i} p_{i}=\sum_{i} w_{i}=$ 1.

In this case, a mapping $F_{p w}: \mathbb{R}^{n} \longrightarrow \mathbb{R}$ is a Weighted Ordered Weighted Averaging (WOWA) operator of dimension $n$ if

$$
F_{p w}\left(a_{1}, a_{2}, \ldots, a_{n}\right)=\sum_{i} \omega_{i} a_{\sigma(i)}
$$

where $\{\sigma(1), \ldots, \sigma(n)\}$ is a permutation of $\{1, \ldots, n\}$ such that $a_{\sigma(i-1)} \geq a_{\sigma(i)}$ for 
all $i \in\{2, \ldots, n\}$ (i.e., $a_{\sigma(i)}$ is the ith largest element in the collection $a_{1}, \ldots, a_{n}$ ) and the weight $\omega_{i}$ is defined as

$$
\omega_{i}=w^{*}\left(\sum_{j \leq i} p_{\sigma(j)}\right)-w^{*}\left(\sum_{j<i} p_{\sigma(j)}\right)
$$

with $w^{*}$ a monotone increasing function that interpolates the points $\left(i / n, \sum_{j \leq i} w_{j}\right)$ together with the point $(0,0) . w^{*}$ is required to be a straight line when the points can be interpolated in this way.

\subsection{Choquet integral}

We will begin defining a fuzzy measure (Sugeno 1974; Wang 1992) in the set of parts of $X, \mathcal{P}(X)$. Grabisch defines (Grabisch 1995):

Definition 3. A function $m: \mathcal{P}(X) \longrightarrow[0,1]$ is a fuzzy measure if and only if it satisfies the following axioms: (i) $m(\emptyset)=0$ and $m(X)=1$ (ii) monotonicity: $B_{1} \subseteq B_{2} \subseteq X$ implies $m\left(B_{1}\right) \leq m\left(B_{2}\right)$.

Among fuzzy measures, some of them are of special interest:

Definition 4. A fuzzy measures $m$ is a Sugeno $\lambda$-measure if $B_{1} \cap B_{2}=\emptyset$ implies that

$$
m\left(B_{1} \cup B_{2}\right)=m\left(B_{1}\right)+m\left(B_{2}\right)+\lambda m\left(B_{1}\right) m\left(B_{2}\right)
$$

for some fixed $\lambda>-1$.

It is proved that for a fixed set of $m\left(\left\{a_{i}\right\}\right), 0<m\left(\left\{a_{i}\right\}\right)<1$, there exists a unique $\lambda \in(-1,+\infty)$ and $\lambda \neq 0$ which satisfies this definition. Then, a Sugeno $\lambda-$ measure is completely determinated.

Grabisch (Grabisch 1995) also define the Choquet integral:

Definition 5. Given a fuzzy measure $m$ the Choquet integral with respect to $m$ can be expressed as:

$$
C h_{m}\left(a_{1} \ldots a_{N}\right)=\sum_{k=1}^{N} a_{\sigma(k)}\left(m\left(A_{\sigma(k)}\right)-m\left(A_{\sigma(k-1)}\right)\right)
$$

where $\{\sigma(1), \ldots \sigma(N)\}$ is a permutation of $\{1, \ldots, N\}$ such that $a_{\sigma(1)} \geq a_{\sigma(2)} \geq$ $\cdots \geq a_{\sigma(N)}, A_{\sigma(k)}=\left\{a_{\sigma(j)} \mid j \leq k\right\}$ (therefore $A_{\sigma(r)}=\left\{a_{\sigma(1)}, \ldots, a_{\sigma(r)}\right\}$ when $r \geq 1$ and $A_{\sigma}(0)=\emptyset$.)

From this definition we can see that weighted means, OWA and WOWA operators are special types of Choquet integral (Torra 1997).

Proposition 1. Let $m$ be a fuzzy measure.

(1) $C h_{m}$ is the weighted mean $M_{p}$ if $m(A)=\sum_{i \in A} p_{i}, \forall A \subseteq X, A \neq \emptyset$.

(2) $C h_{m}$ is the OWA operator $O_{W}$ if $m(A)=\sum_{i=1}^{|A|} w_{i}, \forall A \subseteq X, A \neq \emptyset$.

When the OWA operator is defined by means of a fuzzy quantifier, it is verified that for every monotonically increasing fuzzy quantifier $Q$, we have $O W A_{Q}=C h_{m}$ when $m$ is defined by $m(B)=Q(|B| /|X|), \forall B \subseteq X$. 
(3) For every weighting vector $p$ and every regular monotonically non-decreasing fuzzy quantifier $Q$, we have $W O W A_{Q, p}=C h_{m}$ with $m$ defined by $m_{Q, p}=$ $Q\left(\sum_{a_{i} \in B} p_{i}\right)$ for all $B \subseteq X$.

The last result proves that all WOWA operators are Choquet integrals but not vice versa. A fuzzy measure as this one is said to be a $Q$ - $p$-decomposable fuzzy measure:

Definition 6. A Q-p-decomposable fuzzy measure $m$ is a fuzzy measure $m$ that can be decomposed into a weighting vector $P=\left(p_{1}, p_{2} \ldots p_{N}\right)$ and a monotonically increasing fuzzy quantifier $Q$ so that for all $B \subseteq X$ it is satisfied: $m_{Q, p}(B)=$ $Q\left(\sum_{a_{i} \in B} p_{i}\right)$ is a WOWA

Proposition 2. Any Choquet integral $C_{m}$ with a $Q$-p-decomposable fuzzy measure is a WOWA operator.

Torra also proved that any fuzzy measure that is decomposable by means of a continuous archimedean t-conorm and any Sugeno $\lambda$-measure are $Q$ - $p$-decomposable fuzzy measures.

The rest of the paper is organized as follows: Section 2 analyzes the $L$-fuzzy contexts with multiple weighted values and Section 3 tackles the contexts associated with criteria. Finally, Section 4 analyzes contexts where we have both situations. Conclusions and future work are detailed in Section 5.

\section{2. $\quad L$-fuzzy contexts with multiple values}

In some cases, we have several values representing the relation between an object and an attribute. In the past (Burusco and Fuentes-González 1997) we studied the $L$-fuzzy contexts obtained from several expert opinions to study this situation. In that case multisets were used as a tool for working in that situation without the need of having aggregated observations:

Let $\mathrm{E}$ be a set, and let $\mathcal{M}(E)=\{f: E \longrightarrow \mathbb{N}\}$ be the multisets or bags of $\mathrm{E}$. Let $\mathrm{k} \in \mathbb{N}$ be a finite number; we denoted $M_{k}(E) \subseteq \mathcal{M}(E)$ to the set of multisets of cardinality $k$. We represented a multiset as a collection of elements of $E$ (with repetitions) that corresponds with the non null images of $E$ through $f$.

We were interested in working with the opinion of $k$ experts (set $E$ ), so we reduced the number of observations to $k$ and we work with $M_{k}(L)$.

Definition 7. Let be $L=[0,1]$. An $M(L)$-Fuzzy $k$-valued set $A$ associated to $X$ and an $M(L)$-Fuzzy $k$-valued relation $R$ associated with $X$ and $Y$, are maps $A \in M_{k}(L)^{X}$ and $R \in M_{k}(L)^{X \times Y}$.

Using the theory of expertons (Kaufmann 1988), we defined an order relation $\leq$ in $M_{k}(L)$.

Then, we gave the following definition:

Definition 8. Let be $L=[0,1]$ and let $X$ and $Y$ be the sets of objects and attributes, then the tuple $\left(M_{k}(L), X, Y, R\right)$ is said to be a k-valued L-Fuzzy context.

In that case, we adapted the definition of the derivation operators 1 and 2 to the new situation.

Later (Burusco and Fuentes-González 2001), we analyzed the contexts with multiple weighted values $\left(M_{k}(L), X, Y, E, P, R\right)$ where the sets of experts $E$ and their 
weights $P$ were introduced.

In the first part of the paper, the $M(L)$-fuzzy $k$-valued concepts $(A, B)$ were studied using the theory of expertons:

Definition 9. $A n M(L)$-fuzzy $k$-valued concept $(A, B)$ is a pair with $A \in M_{k}(L)^{X}$ and $B \in M_{k}(L)^{Y}$, verifying $A_{1}=B$ and $B_{2}=A$.

Also in this case, given $A \in M_{k}(L)^{X}$ (or $B \in M_{k}(L)^{Y}$ ), we can obtain the associated $M(L)$-fuzzy $k$-valued concept applying twice the derivation operators. In the case of using a residuated implication, the associated $M(L)$-fuzzy $k$-valued concept is $\left(A_{12}, A_{1}\right)$ (or $\left.\left(B_{2}, B_{21}\right)\right)$.

Let us see an example of that paper:

Example 1. We are interested in the study of the ideal profile to occupy a work place or job. To represent this situation we consult the opinion of 3 personal selection enterprises about the relationship between some courses (objects) and some jobs (attributes). The courses are $X=\{$ computer science, accounting, mechanics, cooking $\}$ and the jobs $Y=\{$ domestic helper, waiter, accountant, car salesman $\}$ and we are also going to suppose that the credibility of the different enterprises is weighted by $p$.

The following table lists the opinion of the three selection enterprises about the relation between courses and jobs:

\begin{tabular}{lcccccccccccccc}
\hline$R$ & \multicolumn{1}{c}{ domestic helper } & \multicolumn{3}{c}{ waiter } & & \multicolumn{3}{c}{ accountant } & \multicolumn{3}{c}{ car salesman } \\
\hline computer science & 0 & 0.2 & 0.5 & 0 & 0.1 & 0.4 & 0.9 & 1 & 1 & 0.4 & 0.4 & 0.8 \\
accounting & 0.2 & 0.3 & 0.8 & 0.3 & 0.3 & 0.6 & 1 & 1 & 1 & 0.7 & 0.3 & 0.7 \\
mechanics & 0 & 0 & 0.3 & 0 & 0.1 & 0.3 & 0 & 0.1 & 0.5 & 0.8 & 0.9 & 1 \\
cooking & 1 & 0.9 & 1 & 0.6 & 0.9 & 1 & 0 & 0 & 0 & 0 & 0 & 0 \\
\hline
\end{tabular}

Firstly, we work with the $M(L)$-fuzzy $k$-valued context. We represent the interest of study by means of an $M(L)$-fuzzy k-valued set and we obtain the corresponding concept.

For instance, if we are interested in computer science and accounting then we take as a starting point the $M(L)$-fuzzy $k$-valued set:

$A=\{$ computer science $/(1,1,1)$, accounting $/(1,1,1)$, mechanics $/(0,0,0)$, cooking $/(0,0,0)\}$

and we obtain the $M(L)$-fuzzy $k$-valued concept that is denoted by $(\hat{A}, \hat{B})$ :

$(\hat{A}, \hat{B})=(\{$ computer science $/(0.8,0.6,0.4)$, accounting $/(0.7,0.7,0.3)$, mechanics $/(0.5,0.1,0)$, $\{$ cooking/(0.1,0,0)\}, domestic helper $/(0.5,0.2,0)$, waiter $/(0.4,0.1,0)$, accountant/ $(1,1,0.9)$, car salesman $/(0.7,0.4,0.3)\})$

The main problem of these concepts is that we lose the association of the different values with the experts. For instance, if the membership degrees are 0.7, 0.3, 0.3 it means that one expert has valued with 0.7 and the others with 0.3 but we are unable to relate each expert to its valuation. By this reason, if we work with $M(L)$-Fuzzy $k$-valued concepts, we can see which is the consensus between the experts but we have the disadvantage that we do not know who has every opinion. Therefore, if we want to aggregate the membership degrees of the $M(L)$-Fuzzy $k$-valued concepts, it is difficult to establish a vector of weights $p$ (for the experts) with different values. What we can only do is to establish $w$ to weight the observations (OWA operator).

Furthermore, in order to obtain a general idea about the $M(L)$-fuzzy $k$-valued 
concept, we can aggregate the different observations for every pair object-attribute. Then, the treatment of aggregation suggested in that work (Burusco and FuentesGonzález 2001) can be improved: we will see in this paper that the use of WOWA operators $F_{p w}$ allows to handle two vectors of weights, one for the observations $(w)$ and the other for the experts $(p)$. We consider that these double weighting vectors allows to obtain results more appropriate to our interest in every moment.

Therefore, to obtain information from an $M(L)$-Fuzzy $k$-valued context, we propose the Multivalued Aggregation Process (MAP):

(1) Calculate the $M(L)$-Fuzzy $k$-valued concepts and aggregate the observations of the degrees of membership of objects and attributes using OWA operators.

(2) Aggregate the observations of the $M(L)$-Fuzzy $k$-valued context using WOWA operators $F_{p w}$ and later obtain the $L$-fuzzy concepts. The new relation $\bar{R} \in$ $L^{X \times Y}$ is defined for every $x \in X$ and $y \in Y$, as follows:

$$
\bar{R}(x, y)=F_{p w}\left(R(x, y)_{1}, R(x, y)_{2}, \ldots, R(x, y)_{k}\right)
$$

with $R(x, y)_{1}, R(x, y)_{2}, \ldots, R(x, y)_{k}$ the $k$ observations associated with the experts $E$ for the pair $(x, y)$.

We can compare these results with that obtained using a weighted mean for context aggregation (Burusco and Fuentes-González 2001). In this case we lose information about the degree of consensus among the different experts but the calculations are simplified. The advantage of using WOWA operators is that they allow us to make a differential treatment of observations and experts.

Example 2. Following the first option of the Multivalued Aggregation Process $(M A P)$, we can aggregate the different values of this initial $M(L)$-Fuzzy $k$-valued concept taking into account that the result can be different depending on the tables of weights that we are using. As the degrees of membership of the objects and attributes come from an experton, we can not associate them with any particular expert. By this reason, as I have commented previously, we apply the WOWA operator with $p=(1 / 3,1 / 3,1 / 3)$ and $w=(3 / 6,2 / 6,1 / 6)$, that is an OWA operator with weight $w=(3 / 6,2 / 6,1 / 6)$.

In that case, we obtain the pair:

(\{computer science/0.7, accounting/0.7, mechanics/0.3, cooking/0\}, $\{$ domestic helper/0.3, waiter/0.2, accountant/1, car salesman/0.5\})

that can be interpreted saying that computer science and accounting are the most important courses for the job of accountant and in a much lesser degree for car salesman.

Remark 1. The pair obtained pair is not necessarily an $L$-fuzzy concept of the $L$-fuzzy context $(L, X, Y, R)$. Furthermore, we obtain different results with the election of different weighting vectors for $F_{p w}$.

The other option of the Multivalued Aggregation Process (MAP) consists of aggregating the values of the $M(L)$-Fuzzy $k$-valued context by means of an WOWA operator. We use the vector $w=(3 / 6,2 / 6,1 / 6)$ since we want to give more relevance to the largest membership degrees. Also two different weighting vectors for the experts are chosen depending on our opinion about the companies:

- $p_{1}=(1 / 6,2 / 6,3 / 6)$. The degree of credibility of the enterprises are growing from the first one to the last one.

The result of the aggregation process using $F_{p_{1} w}$ is: 


\begin{tabular}{lcccc}
\hline $\bar{R}_{1}$ & domestic helper & waiter & accountant & car salesman \\
\hline computer science & 0.4 & 0.3 & 1 & 0.7 \\
accounting & 0.6 & 0.5 & 1 & 0.6 \\
mechanics & 0.2 & 0.2 & 0.4 & 0.9 \\
cooking & 1 & 0.9 & 0 & 0 \\
\hline
\end{tabular}

- $p_{2}=(0.4,0.4,0.2)$. We are giving a small weight to the third company.

In this case, using $F_{p_{2} w}$ we obtain:

\begin{tabular}{lcccc}
\hline $\bar{R}_{2}$ & domestic helper & waiter & accountant & car salesman \\
\hline computer science & 0.2 & 0.1 & 0.9 & 0.5 \\
accounting & 0.4 & 0.4 & 1 & 0.6 \\
mechanics & 0.1 & 0.1 & 0.3 & 0.9 \\
cooking & 1 & 0.8 & 0 & 0 \\
\hline
\end{tabular}

The third company has valued the relationship between courses and jobs in a more positive way, hence when we have given a smaller weight, as in this case, the resulting values are smaller than the previous ones.

In addition, to compare these two contexts each other it would be interesting to compare them with the obtained adding the initial context with the weighted mean values using $p_{1}=(1 / 6,2 / 6,3 / 6)$ and $p_{2}=(0.4,0.4,0.2)$, as we did in the previous work (Burusco and Fuentes-González 2001).

In the case of aggregating using $F_{p_{1} w}$, we have:

\begin{tabular}{lcccc}
\hline $\bar{R}_{3}$ & domestic helper & waiter & accountant & car salesman \\
\hline computer science & 0.3 & 0.2 & 1 & 0.6 \\
accounting & 0.5 & 0.4 & 1 & 0.6 \\
mechanics & 0.2 & 0.2 & 0.3 & 1 \\
cooking & 1 & 0.9 & 0 & 0 \\
\hline
\end{tabular}

and with $F_{p_{2} w}$ :

\begin{tabular}{lcccc}
\hline $\bar{R}_{4}$ & domestic helper & waiter & accountant & car salesman \\
\hline computer science & 0.2 & 0.1 & 1 & 0.5 \\
accounting & 0.4 & 0.4 & 1 & 0.5 \\
mechanics & 0.1 & 0.1 & 0.1 & 0.9 \\
cooking & 1 & 0.8 & 0 & 0 \\
\hline
\end{tabular}

In general, we can say that membership degrees of the relationships obtained by WOWA operators $\left(\bar{R}_{1}\right.$ and $\left.\bar{R}_{2}\right)$ are larger than those obtained by aggregation using a weighted mean with $p_{1}$ and $p_{2}\left(\bar{R}_{3}\right.$ and $\left.\bar{R}_{4}\right)$. The reason is that we are using vectors $w$ that give more relevance to the largest observations.

In particular, if we want to study the course of mechanics, for example, we take as a starting set $\{$ computer science/0, accounting/0, mechanics/1, cooking/0\} and obtain the result:

- Using relation $R_{2}$ (WOWA):

(\{computer science/0.6, accounting/0.7, mechanics/1, cooking/0.1\},

\{domestic helper/0.1, waiter/0.1, accountant/0.3, car salesman/0.9\})

- Using relation $R_{4}$ (Weighted mean): 
( $\{$ computer science/0.6, accounting/0.6, mechanics/1, cooking/0.1\},

$\{$ domestic helper/0.1, waiter/0.1, accountant/0.1, car salesman/0.9\})

The first $L$-fuzzy concept notes that accountant $\left(y_{3}\right)$ is related to mechanics $\left(x_{3}\right)$, although to a lesser degree. This aspect, which was not observed with the weighted mean and that corresponds to the initial information, appears using WOWA operators.

We can conclude by saying that to get a more complete information of the $M(L)$ Fuzzy $k$-valued context, we can aggregate the values. The WOWA operators allow to make a better and more complete study of the $M(L)$-Fuzzy $k$-valued contexts, particularly when we aggregate the initial context observations previously to calculate $L$-fuzzy concepts. These operators allow to use two weighting vectors, one for the experts and the other for the values. Then, we can establish certain nuances that respond to our interests.

\section{3. $\quad L$-fuzzy contexts associated with criteria}

Sometimes, we are interested in the study of the relationship between objects and attributes from different points of view (criteria). We defined the $L$-fuzzy $C$-contexts as a model to represent these kind of situations (Burusco and Fuentes-González 2002). The contexts were defined as follows:

Definition 10. Let be $L=[0,1]$, let $X, Y$ and $C$ be non-empty and finite sets of objects, attributes and criteria, and $R \in L^{X \times Y}$ an $L$-fuzzy relation. The tuple $(L, X, Y, R, C)$ is said to be the $L$-fuzzy $C$-context. The cardinality of $X, Y$ and $C$ is denoted by $n, m$ and $l$, respectively.

Let us see an example (Burusco and Fuentes-González 2002):

Example 3. Suppose that there are some training courses for unemployed that want to find a job. To represent this situation we take the $L$-fuzzy $C$-context $(L, X, Y, R, C)$ where $L=[0,1]$, the object set $X=\{$ computer science, accounting, carpentry, cooking, music, painting $\}$ is the set of training courses, the attribute set $Y=\{$ domestic helper, waiter, accountant, furniture salesman, carpenter, insurance agent $\}$ is the set of jobs, $C=\{$ great difficulty, few hours, cheap, very general, practical $\}$ contains the different criteria to value the courses, and the relation $R$ represents a possible relationship between the training courses and the offered jobs.

\begin{tabular}{|c|c|c|c|c|c|c|}
\hline$R$ & dom. helper & waiter & account. & furni. sales. & carpenter & insur. agent \\
\hline comp. sci. & 0 & 0.2 & 0.8 & 0.2 & 0.2 & 0.5 \\
\hline accounting & 0 & 0.2 & 1 & 0.2 & 0 & 0.6 \\
\hline carpentry & 0 & 0 & 0 & 0.6 & 1 & 0 \\
\hline cooking & 1 & 0.6 & 0 & 0 & 0 & 0 \\
\hline music & 0 & 0.4 & 0 & 0 & 0 & 0 \\
\hline painting & 0.4 & 0 & 0 & 0.2 & 0.8 & 0 \\
\hline
\end{tabular}

In this case, the derivation operators are defined using a fuzzy implication operator $I$ as follows:

Definition 11. For every relation $F \in L^{C \times X}$ we can associate an element $F_{1}$ of $L^{C \times Y}$ :

$$
F_{1}(c, y)=\inf _{x \in X}\{I(F(c, x), R(x, y))\}
$$


In the same way, for every $G \in L^{C \times Y}$ we can associate $G_{2} \in L^{C \times X}$ :

$$
G_{2}(c, x)=\inf _{y \in Y}\{I(G(c, y), R(x, y))\}
$$

We proved (Burusco and Fuentes-González 2002) that the $L$-fuzzy $C$-concepts are pairs $(\hat{F}, \hat{G})$ with $\hat{F} \in L^{C \times X}$ and $\hat{G} \in L^{C \times Y}$ such that applying the derivation operator to one of the relations we get the other one. The interpretation of each concept is based on different criteria (rows of the corresponding matrices that form the pair). The set of the $L$-fuzzy $C$-concepts is a complete lattice.

Moreover, we also proved that if we take the $L$-fuzzy concept $(\hat{F}, \hat{G})$ of the $L$-fuzzy $C$-context $(L, X, Y, R, C)$, for any $c \in C$ we have $\left(\hat{F}_{c}, \hat{G}_{c}\right)$ an $L$-fuzzy concept of the $L$-fuzzy context $(L, X, Y, R)$.

Let us see an example that appears in(Burusco and Fuentes-González 2002)

Example 4. Suppose that the following table $F \in L^{C \times X}$ represents the opinion of an expert about the different courses (objects) taking into account some concrete aspects (criteria).

\begin{tabular}{lcccccc}
\hline F & comp. science & accounting & carpentry & cooking & music & painting \\
\hline great difficulty & 1 & 1 & 0 & 0 & 0 & 0 \\
few hours & 0 & 0 & 0 & 1 & 0 & 0 \\
cheap & 0 & 0 & 1 & 0 & 0 & 0 \\
very general & 0 & 0 & 1 & 0 & 0 & 1 \\
practical & 1 & 1 & 0.5 & 0.5 & 0 & 0 \\
\hline
\end{tabular}

We can obtain the derivated L-fuzzy $C$-concept. This is the resulting pair $(\hat{F}, \hat{G})$ calculated in Burusco and Fuentes-González (2002) using the Kleene-Dienes implication operator.

\begin{tabular}{lcccccc}
\hline$\hat{F}$ & comp. science & accounting & carpentry & cooking & music & painting \\
\hline great difficulty & 0.5 & 0.6 & 0.2 & 0.2 & 0.2 & 0.2 \\
few hours & 0.4 & 0.4 & 0.4 & 0.6 & 0.4 & 0.4 \\
cheap & 0.2 & 0.2 & 0.6 & 0.2 & 0.2 & 0.4 \\
very general & 0.2 & 0.2 & 0.8 & 0.2 & 0.2 & 0.8 \\
practical & 0.5 & 0.6 & 0.5 & 0.5 & 0.5 & 0.5 \\
\hline
\end{tabular}

\begin{tabular}{lcccccc}
\hline$\hat{G}$ & dom. helper & waiter & account. & furni. sales. & carpenter & insur. agent \\
\hline great diffic. & 0.4 & 0.4 & 0.8 & 0.4 & 0.4 & 0.5 \\
few hours & 0.6 & 0.6 & 0.4 & 0.4 & 0.4 & 0.4 \\
cheap & 0.4 & 0.4 & 0.4 & 0.6 & 0.8 & 0.4 \\
very general & 0.2 & 0.2 & 0.2 & 0.2 & 0.8 & 0.2 \\
practical & 0.4 & 0.4 & 0.5 & 0.4 & 0.4 & 0.5 \\
\hline
\end{tabular}

To understand the meaning of this concept, we have to take into account that each object or attribute have different membership values associated with the different criteria. Then, for every criteria, we take those objects and attributes with the largest membership degrees.

For instance, in this case we can say that if the unemployed is interested in a cheap course, then he will study carpentry to work as a carpenter or furniture salesman. 
Another important point is to get an overview of the $L$-fuzzy $C$-concept. We will proceed in a different way depending on the dependence or independence of the criteria.

\subsection{Study with independent criteria}

As we have shown, the starting point is the $L$-fuzzy $C$-concept $(\hat{F}, \hat{G})$ derived from the opinion $F \in L^{C \times X}$ about the degree in which every object (or attribute) verifies the different criteria. We also take into account the relationship between the objects and the attributes. A similar process can be done taking as a starting point an $L$ fuzzy set of attributes $G \in L^{C \times Y}$.

In order to obtain a more complete information about the $L$-fuzzy $C$-concepts, we can aggregate the rows of each one of the relations that form the concept. It is at this point where WOWA operators can be involved when the criteria are independent. Again, vector $w$ is used to weight the observations and vector $p$ to weight the criteria. Specifically, we are interested in the observations with the largest membership degrees that stand out in our $L$-fuzzy concepts. We will try to get them using vector $w$.

Furthermore, a ranking of objects and attributes can be established.

Definition 12. Let be $|C|=l$. Given the L-Fuzzy $C$-concept $(\hat{E}, \hat{F})$ and $F_{p w} a$ WOWA operator of dimension $l$ associated with the weighting vectors $p$ and $w$. We define the pair $(\bar{F}, \bar{G}) \in L^{X} \times L^{Y}$ as follows:

$$
\begin{aligned}
& \bar{F}(x)=F_{p w}\left(\hat{F}\left(c_{1}, x\right), \hat{F}\left(c_{2}, x\right), \ldots \hat{F}\left(c_{l}, x\right)\right), \text { for all } x \in X \\
& \bar{G}(y)=F_{p w}\left(\hat{G}\left(c_{1}, y\right), \hat{G}\left(c_{2}, y\right), \ldots \hat{G}\left(c_{l}, y\right)\right), \text { for all } y \in Y
\end{aligned}
$$

Remark 2. The pair $(\bar{F}, \bar{G}) \in L^{X} \times L^{Y}$ is not necessarily an $L$-fuzzy concept of the $L$-fuzzy context $(L, X, Y, R)$. Furthermore, we obtain different results with the election of different weighting vectors for $F_{p w}$.

Now, we are going to define an order relation for the elements of $X$ and $Y$.

Definition 13. Given $(\bar{F}, \bar{G}) \in L^{X} \times L^{Y}$ the pair resulting of the aggregation process. For every $x_{i}, x_{j} \in X$,

$$
x_{i} \preceq x_{j} \text { if } \bar{F}\left(x_{i}\right) \leq \bar{F}\left(x_{j}\right)
$$

Analogously for the attributes $Y$.

This is a preorder relation that allows to establish the Object and the Attribute Rankings associated with $(\bar{F}, \bar{G})$.

The use of WOWA operators $F_{p w}$ with a double vector of weights, $p$ for the criteria and another $w$ for the membership degrees of the observations allows to establish different nuances that otherwise would not be possible. We can see this aspect below:

Example 5. We can come back to consider the L-fuzzy $C$-concept $(\hat{F}, \hat{G})$ derived from the opinion of an expert, that represents those combinations of courses and jobs associated with each criterion. 
For the aggregation, we take the WOWA operator $F_{p w}$ with weights $p_{i}=1 / 5, \forall i \in$ $\{1, \ldots, 5\}$, that is, the student thinks that all the criteria are equally important and $w=(5 / 15,4 / 15,3 / 15,2 / 15,1 / 15)$ to give more relevance to the largest values. Then the obtained result is:

$\bar{F}=\{$ computer science $/ 0.42$, accounting $/ 0.48$, carpentry $/ 0.59$, cooking $/ 0.41$, music/0.35, painting/0.55\}

$\bar{G}=\{$ domestic helper $/ 0.45$, waiter $/ 0.45$, accountant $/ 0.55$, furniture salesman $/ 0.45$, carpenter $/ 0.64$, insurance agent $/ 0.45\}$

Taking into account Definition 13, and ordering the membership degrees in an increasing way, we can establish different rankings for objects (ranking of courses), and for attributes (ranking of jobs) (See Figure 1).
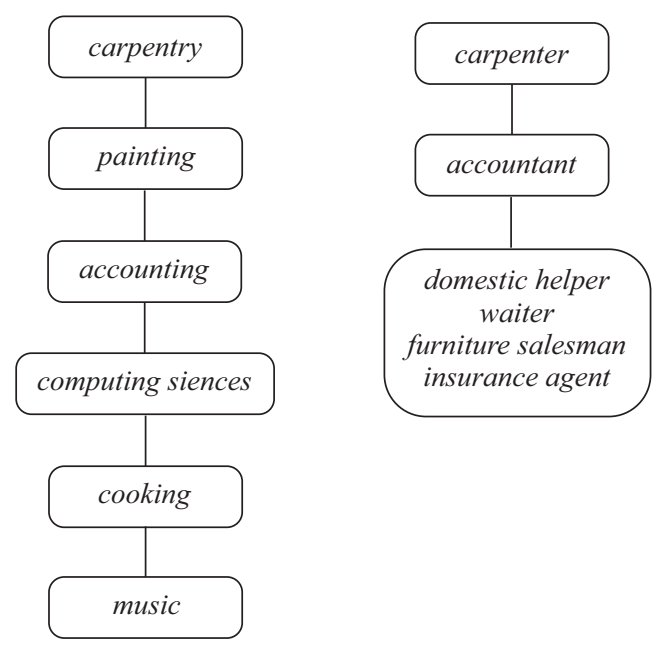

Figure 1. Rankings of courses and jobs

That means that if all the criteria are equally relevant for the student, he must begin studying "carpentry", hereafter "painting", "accounting", "computer science", "cooking", and finally "music". On the other hand, the jobs suitable for this person are, in the first place, "carpenter", after "accountant" and the other ones in the last place.

However, the result is different if we use another weighting vector $w$. For instance, $w=(1,0,0,0,0)$ provides the rankings shown in Figure 2:
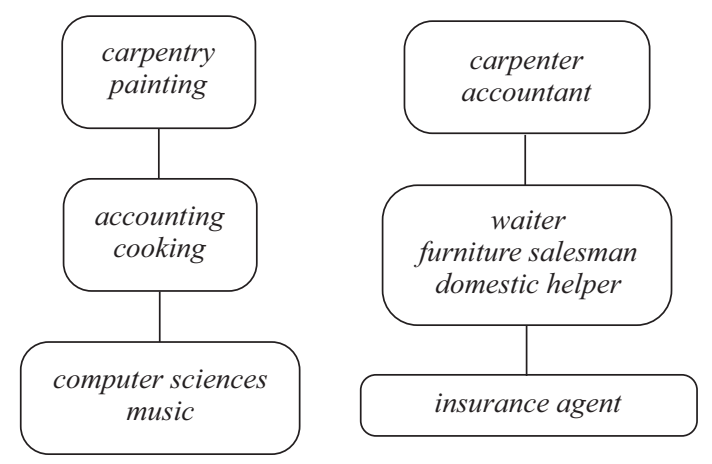

Figure 2. Rankings obtained for $w=(1,0,0,0,0)$

With this election of vector $w$ we want to take into account only the biggest membership degrees. For this reason, the result changes. 
Now, we are going to see what happens if a student considers that some criteria are more important than the others. For instance, we suppose that he thinks that the most important thing for a course is to be "short" and "practical". To model this situation, we are going to use WOWA operators and take the following weights $p=(0,3 / 5,0,0,2 / 5)$ and $w=(5 / 15,4 / 15,3 / 15,2 / 15,1 / 15)$. The result is:

$\bar{F}=\{$ comp. sciences $/ 0.46$, accounting/0.52, carpentry/0.46, cooking/0.58, music/0.46, painting/0.46\}

$\bar{G}=\{$ domestic helper $/ 0.56$, waiter $/ 0.56$, accountant $/ 0.46$, furniture salesman $/ 0.4$, carpenter $/ 0.4$, insurance agent/0.46\}

Therefore, we obtain a different order for the previous situation, as can be seen in Figure 3:

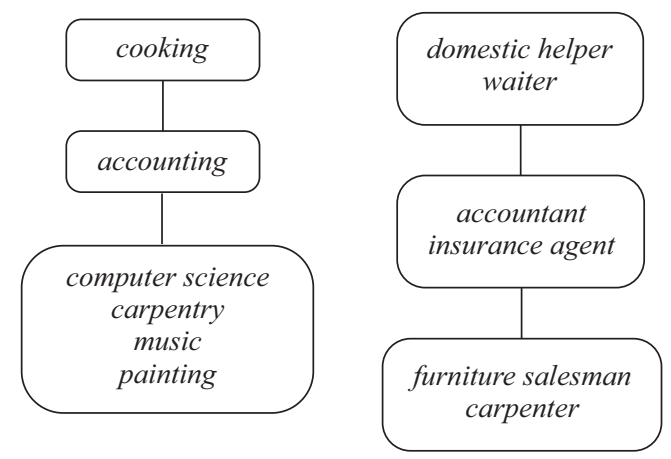

Figure 3. Rankings obtained using WOWA operators

We can see that the jobs directly related to the "cooking" course appear in the first place and those associated with the "accounting" later.

Also in this case, the election of a different vector $w$ changes the result. With the same $p=(0,3 / 5,0,0,2 / 5)$ and $w=(1,0,0,0,0)$ we obtain the rankings represented in Figure 4:

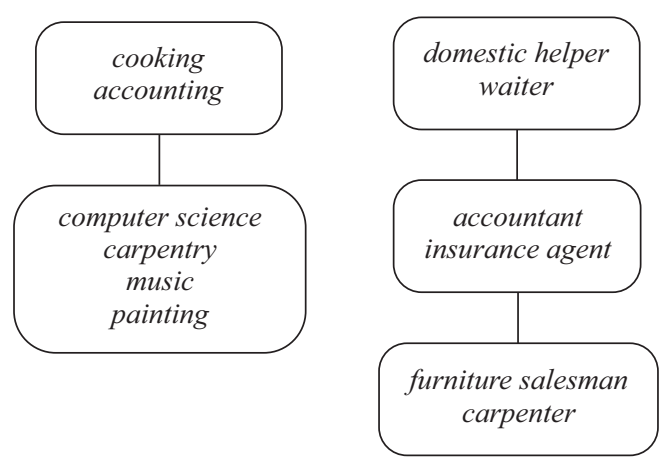

Figure 4. Rankings obtained taking $w=(1,0,0,0,0)$ in the WOWA operator

The classification obtained in both cases using WOWA operators is the same as the one obtained in the previous work (Burusco and Fuentes-González 2002) if we use $w=(5 / 15,4 / 15,3 / 15,2 / 15,1 / 15)$, although membership degrees of objects and attributes are not preserved. The fact that we have used WOWA operators which give more relevance to observations closest to one has allowed to obtain concepts with objects and attributes that have larger membership degrees in all cases. I.e. through the use of $w$ we have nuanced the result taking the greatest values.

However, the result changes if we use, for instance, $w=(1,0,0,0,0,0)$. 


\subsection{Study with dependent criteria}

We have defined the $L$-fuzzy $C$-contexts as a model to represent the relationship between objects and attributes from different points of view (criteria).

In order to obtain a general information from these $L$-fuzzy $C$-concepts, in the previous section we have assumed that the criteria were independent. If the criteria are dependent then a group of criteria can give a better result if they are combined instead of being treated in isolation. In (Burusco and Fuentes-González 2002) we represented these situations by means of rules. In that case, a t-norm was used in order to evaluate the antecedent of the rules.

Another more natural possibility will be to establish the dependence among the criteria by means of the expert opinion $F \in L^{C \times X}$ about the relation between the criteria and the objects. With this aim, we propose the use of Choquet integrals to aggregate the values of the $L$-fuzzy $C$-concept. It is important to highlight that the use of Choquet integrals allows to set up aggregations for situations where an object belongs to several groups (in this case, a classical aggregation will not be useful).

Next, we will describe the process from the point of view of the objects. Another similar one, from the point of view of the attributes, can be performed.

Firstly, we introduce some necessary definitions:

Given $F \in L^{C \times X}$ that represents the opinion of an expert about the relation between the criteria and the objects, we can define the $L$-fuzzy context $(L, C, X, F)$. In this case, the object set is $C$ and the attribute set $X$. Then, for every $c_{i} \in C, i \in$ $\{1, \ldots, l\}$, let $\mathbf{c}_{\mathbf{i}}$ be such that $\mathbf{c}_{\mathbf{i}}\left(c_{i}\right)=1$ and $\mathbf{c}_{\mathbf{i}}(c)=0$, for any $c \neq c_{i}$. We denote the concepts derived from the different $\mathbf{c}_{\mathbf{i}}, i=\{1, \ldots, n\}$, by $\mathcal{C}_{\mathbf{i}}$.

For every $L$-fuzzy concept $\mathcal{C}$ of $(L, C, X, F)$, we denote the membership degree of the object $c_{i}$ to the extension of $\mathcal{C}$ by $\operatorname{memb}\left(c_{i}, \mathcal{C}\right)$.

Definition 14. Let be $0<\alpha \leq 1$. For every $L$-fuzzy concept $\mathcal{C}$ of the $L$-fuzzy context $(L, C, X, F)$, we define the $\alpha$-objects associated with $\mathcal{C}$ as the set $T_{\mathcal{C}}^{\alpha}=\left\{c_{i} \in C \mid\right.$ memb $\left.\left(c_{i}, \mathcal{C}\right) \geq \alpha\right\}$ Analogously, the $\alpha$-attributes associated with $\mathcal{C}$ can be defined.

Definition 15. If $c_{k} \in T_{\mathcal{C}_{\mathrm{i}}}^{\alpha}, k \neq i$, then $c_{i}$ is said to be $\alpha$-dependent on $c_{k}$. On the contrary, we say that $c_{i}$ is $\alpha$-independent on $c_{k}$.

That is, $c_{i}$ is $\alpha$-dependent on $c_{k}$ when in the $\mathcal{C}_{\mathbf{i}}$ derived $L$-fuzzy concept, $c_{k}$ stands out in a level bigger than or equal to $\alpha$. In the case $\alpha=1$, we have that the membership degree of $c_{k}$ in the $L$-fuzzy concept is equal to 1 .

Definition 16. Given $\alpha$, we say that $c_{i}$ is $\alpha$-independent if there is no $c_{k}$ such that $c_{k} \in T_{\mathcal{C}_{\mathrm{i}}}^{\alpha}, k \neq i$.

Example 6. Taking as a starting point the opinion of the expert $F \in L^{C \times X}$ of the previous example, we obtain the following result represented in Figure 5 when $\alpha=1$ :

$T_{\mathcal{C}_{1}}^{1}=\left\{c_{1}, c_{5}\right\}, T_{\mathcal{C}_{2}}^{1}=\left\{c_{2}\right\}, T_{\mathcal{C}_{3}}^{1}=\left\{c_{3}, c_{4}\right\}, T_{\mathcal{C}_{\mathbf{c}_{4}}}^{1}=\left\{c_{4}\right\}, T_{\mathcal{C}_{5}}^{1}=\left\{c_{5}\right\}$.

When $\alpha=0.5$ the obtained result is the following one (See Figure 6): $T_{\mathcal{C}_{1}}^{0.5}=$ $\left\{c_{1}, c_{5}\right\}, T_{\mathcal{C}_{2}}^{0.5}=\left\{c_{2}, c_{5}\right\}, T_{\mathcal{C}_{3}}^{0.5}=\left\{c_{3}, c_{4}, c_{5}\right\}, T_{\mathcal{C}_{4}}^{0.5}=\left\{c_{4}\right\}, T_{\mathcal{C}_{5}}^{0.5}=\left\{c_{1}, c_{5}\right\}$.

Now we take the $L$-Fuzzy $C$-concept $(\hat{F}, \hat{G})$ derived from $F \in L^{C \times X}$ and, in order to establish a summarized information, we can use the following Criteria Aggregation Process (CRP):

(1) For every $c_{i}, i \in\{1, \ldots, l\}$ we obtain its derived $L$-fuzzy concept $\mathcal{C}_{\mathbf{i}}$ in the $L$-fuzzy context $(L, C, X, F)$.

(2) For every $\mathcal{C}_{\mathbf{i}}, i \in\{1, \ldots, l\}$ and given $0<\alpha \leq 1$, we define the set $T_{\mathcal{C}_{\mathbf{i}}}^{\alpha}$ of 


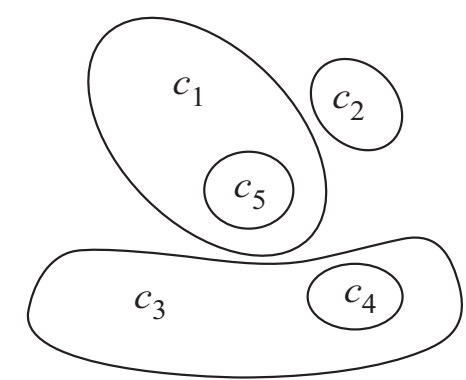

Figure 5. Grouping of criteria if $\alpha=1$

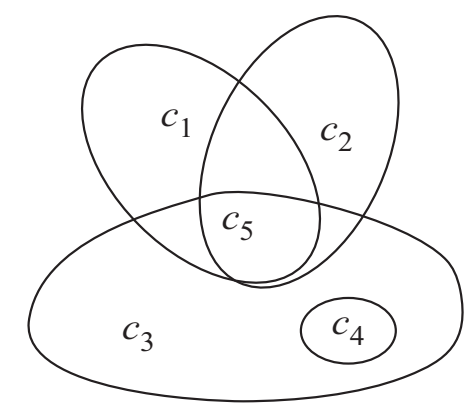

Figure 6. Grouping of criteria if $\alpha=0.5$

$\alpha$-objects associated with $\mathcal{C}_{\mathbf{i}}$.

(3) We select the maximum value $\hat{\alpha}$ such that all the sets $T_{\mathcal{C}_{\mathbf{i}}}^{\hat{\alpha}}$ are pairwise non disjoint sets. These sets $T_{\mathcal{C}_{\mathrm{i}}}^{\hat{\alpha}}$ will be used to define a measure $m$. (We will use the measure to give more relevance to the criteria associated with the same $\left.T_{\mathcal{C}_{\mathrm{i}}}^{\hat{\alpha}}\right)$

(4) We aggregate the rows of the $L$-Fuzzy $C$-concept $(\hat{F}, \hat{G})$ associated with the different $c_{i}, i \in\{1, \ldots, l\}$ using the Choquet integral associated with $m$. The result is a pair of $L$-fuzzy sets $\left(\bar{F}^{\hat{\alpha}}, \bar{G}^{\hat{\alpha}}\right) \in L^{X} \times L^{Y}$ :

$\bar{F}^{\hat{\alpha}}(x)=C h_{m}\left(F\left(c_{1}, x\right), F\left(c_{2}, x\right), \ldots F\left(c_{l}, x\right)\right)$, for all $x \in X$

$\bar{G}^{\hat{\alpha}}(y)=C h_{m}\left(G\left(c_{1}, y\right), G\left(c_{2}, y\right), \ldots G\left(c_{l}, y\right)\right)$, for all $y \in Y$

with $C h_{m}$ the Choquet integral with respect to $m$ and $c_{i} \in C, i \in\{1, \ldots, l\}$

Remark 3. As in previous cases, the pair $(\bar{F}, \bar{G}) \in L^{X} \times L^{Y}$ is not necessarily an $L$-fuzzy concept of the $L$-fuzzy context $(L, C, X, F)$.

We will take into account the Criteria Aggregation Process (CRP) (step (3)) in order to obtain $\hat{\alpha}$.

Then, the measure $m$ for the Choquet integral is defined, for every $c_{i}, i \in$ $\{1, \ldots, l\}$, as follows:

$$
m\left(c_{i}\right)=\left\{\begin{array}{l}
2 / l \text { if } c_{i} \text { is } \hat{\alpha} \text {-independent } \\
1 / l \text { otherwise }
\end{array}\right.
$$

where $a=2 / l$ and $b=1 / l$ with $l$ the cardinality of $C$.

And, for the rest of the sets:

$m\left(\cup_{i \in I}\left(c_{i}\right)\right)=\left\{\begin{array}{l}\min \left(\sum_{i \in I} m\left(c_{i}\right)+1 / l, 1\right) \text { if it exists an } \hat{\alpha} \text {-dependent } k \in I, \text { such that } \underset{i \in I}{\cup}\left(c_{i}\right) \supseteq T_{\mathcal{C}_{\mathbf{k}}}^{\hat{\hat{\alpha}}} \\ \min \left(\sum_{i \in I} m\left(c_{i}\right), 1\right) \text { otherwise }\end{array}\right.$ 
Example 7. As can be seen in the previous example, $\hat{\alpha}=0.5, m\left(c_{1}\right)=m\left(c_{2}\right)=$ $m\left(c_{3}\right)=m\left(c_{5}\right)=0.2$ and $m\left(c_{4}\right)=0.4$. Following the proposed measure definition, we can apply the Choquet integral in order to aggregate the values corresponding to the different criteria for the objects and for the attributes. The result is $\bar{F}^{\hat{\alpha}}$ and $\bar{G}^{\hat{\alpha}}$ : $\bar{F}^{\hat{\alpha}}=\{$ comp.science $/ 0.42$, accounting/0.48, carpentry $/ 0.62$, cooking $/ 0.4$, music $/ 0.34$, carpentry $/ 0.58\}$ $\bar{G}^{\hat{\alpha}}=\{$ domes.helper/0.44, waiter/0.44, account./0.52, furnit.sales/0.44, carpen./0.56, insur.agent/0.46 $\}$

Therefore, taking into account the opinion of the expert and Definition 13, we can establish the Object and Attribute Rankings represented in Figure $\%$.

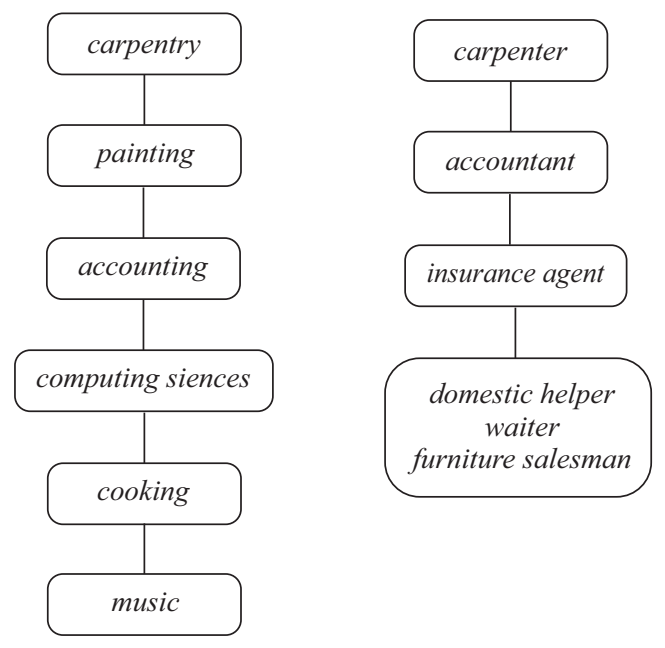

Figure 7. Object and Attribute Rankings

\section{Multivalued $L$-fuzzy contexts associated with criteria}

At this point, we are interested in addressing the situation where we have an $L$ fuzzy context associated with criteria where we have several values to represent the relationship between and object and an attribute. That is, the study of the relationship between a set of objects $X$ and a set of attributes $Y$ from different points of view $C$ (criteria) representing the opinion of different experts $E$.

The framework is the following:

Definition 17. Let $X, Y, E$ and $C$ be sets of cardinality $n, m, k$ and $l$ respectively. We define an $M(L)$-fuzzy $k$-valued $C$-context as a tuple $\left(M_{k}(L), X, Y, R, E, C\right)$ where $L=[0,1], X$ is the set of objects, $Y$ the set of attributes, $C$ the set of criteria, $E$ the set of experts and $R \in M_{k}(L)^{X \times Y}$.

At this point, three different studies can be perform depending on our interest:

(1) If the criteria are not very important for us, we can dispense with them working with the $M(L)$-fuzzy $k$-valued context $\left(M_{k}(L), X, Y, R\right)$ as in Section 2 (dispense with $C$ set).

(2) For every expert $e \in E$, another possibility is working with the $L$-fuzzy $C$ context $\left(L, X, Y, R_{e}, C\right)$, where $R_{e}(x, y)=R(x, y)_{e}$, for all $x \in X, y \in Y$ is the value corresponding with the expert $e$ in the relation $R \in M_{k}(L)^{X \times Y}$. At this point, we can proceed as in Section 3.

(3) Finally, we can be interested in working both with the criteria and with the experts. We can adapt the derivation operators 1 and 2, and the definition of 
$L$-fuzzy concepts to this new situation. However, we consider that the process is too complex. For this reason, when we are interested in a general overview, our recommendation is the use of aggregation tools: WOWA operators for the different observations of every pair object-attribute and also Choquet integral when we have to interpret the meaning of the obtained $L$-fuzzy $C$-concepts. Then, we will transform the $M(L)$-fuzzy $k$-valued context associated with criteria $\left(M_{k}(L), X, Y, R, E, C\right)$ into an $L$-fuzzy $C$-context $(L, X, Y, \bar{R}, C)$. The new relation $\bar{R}$ is obtained using a WOWA operator $F_{p w}$ associated with the weighting vectors $p$ and $w$.

$$
\bar{R}(x, y)=F_{p w}\left(R(x, y)_{1}, R(x, y)_{2}, \ldots, R(x, y)_{k}\right) \text {, for all } x \in X, \text { for all } y \in Y
$$

with $R(x, y)_{1}, R(x, y)_{2}, \ldots, R(x, y)_{k}$ the $k$ observations associated with the experts $E$ for the pair $(x, y)$.

\section{Conclusions and future work}

In this work, we have used WOWA operators and Choquet integrals as aggregation tools for the $L$-fuzzy contexts study when $L=[0,1]$ in two different situations:

(1) In contexts that represent the opinion of multiple experts about the relationship between the objects and the attributes in an $L$-fuzzy context.

(2) When we are interested in the study of $L$-fuzzy contexts taking into account different criteria.

In both cases we have extended the results obtained in previous works. Then, we can say that this new approach allows us to make a more complete study of the different $L$-fuzzy contexts. In this sense, we have improved the extraction of knowledge using these tools in the $L$-fuzzy concept analysis.

As a future work, we want to extend these results to any complete lattice $L$. We are also interested in the use of penalty functions in order to improve the aggregation processes.

\section{Acknowledgments}

This paper is partially supported by the Research Group "Intelligent Systems and Energy (SI+E)" of the Basque Government, under Grant IT677-13 and by the Research Group "Artificial Intelligence and Approximate Reasoning" of the Public University of Navarra, under TIN2016-77356-P (MINECO, AEI/FEDER, UE).

\section{References}

Alcalde, C., Burusco, A., Fuentes-González, R. and Zubia, I. 2009. "Treatment of L-fuzzy contexts with absent values." Information Sciences 179 (1-2):1-15.

Alcalde,C., Burusco, A., Bustince, H., Jurío, A. and Sanz, J.A. 2016. "Evolution in time of the $L$-fuzzy context sequences." Information Sciences 326:202-214.

Bělohlávek, R. 1999. "Fuzzy Galois Connections." Math. Logic Quarterly 45 (4):497-504.

Bělohlávek, R. and Vychodil, V. 2005. "What is a fuzzy concept lattice." In Proceedings 
of the Third International Conference on Concept Lattice and their Applications, CLA 2005 Olomounc, Czech Republic 34-45.

Burusco, A. and Fuentes-González, R. 1994. "The Study of the L-Fuzzy Concept Lattice." Mathware and Soft Computing 1 (3):209-218.

Burusco, A. and Fuentes-González, R. 1998. "Construction of the L-Fuzzy Concept Lattice." Fuzzy Sets and Systems 97 (1):109-114.

Burusco, A. and Fuentes-González, R. 1997. "Contexts obtained from several expert opinions. Sixth IEEE International Conference on Fuzzy Systems, Barcelona 339-342.

Burusco, A., Fuentes-González, R. 2001. "Contexts with multiple weighted values. The International Journal of Uncertainty, Fuzziness and Knowledge-based Systems 9 (3):355368.

Burusco, A. and Fuentes-González, R. 2001. "The study of the interval-valued contexts" Fuzzy Sets and Systems 121:69-82.

Burusco, A. and Fuentes-González, R. 2002. "Concepts associated to criteria: a method for knowledge processing from fuzzy contexts" The International Journal of Uncertainty, Fuzziness and Knowledge-based Systems 10 (2):173-184.

Calvo, T. and Mesiar, R. 2003. "Weighted triangular norms-based aggregation operators." Fuzzy Sets and Systems 137:3-10.

Calvo, T. and Mesiar, R. 2003. "Aggregation operators: ordering and bounds." Fuzzy Sets and Systems 139:685-697.

Djouadi, Y. and Prade, H. 2010. "Interval-Valued Fuzzy Galois Connections: Algebraic Requirements and Concept Lattice Construction.", Fundamenta Informaticae 99 (2):169186.

Djouadi, Y. and Prade, H. 2011. "Possibility-theoretic extension of derivation operators in formal concept analysis over fuzzy lattices.", FODM 10 (4) 287-309.

Fodor, J. and Roubens, M. 1994. "Fuzzy Preference Modelling and Multicriteria Decision Support." In Theory and Decision Library Kluwer Academic Publishers.

Grabisch, M. 1995. Fuzzy integral in multicriteria decision making, Fuzzy Sets and Systems 69 (3): 279-298.

Kaufmann, A. 1988. "Theory of expertons and fuzzy logic." Fuzzy Sets and Systems 28:295304.

Medina, J. and Ojeda-Aciego, M. 2010. "Multi-adjoint t-concept lattices." Information Sciences 180 (5):712-725.

Medina, J. 2012. "Multi-adjoint property-oriented concept lattices." Information Sciences 190:95-106.

Medina, J. and Ojeda-Aciego, M. 2013. "Dual multi-adjoint concept lattices." Information Sciences 225:47-54.

Pollandt, S. 1997. Fuzzy Begriffe Springer.

Sugeno, M. 1974. Theory of Fuzzy Integrals and its Applications, (PhD Dissertation). Tokyo Institute of Technology, Tokyo, Japan, (1974).

Torra, V. 1997. "The weighted OWA Operator." International Journal of Intelligent Systems 12:153-166.

Wang, Z., Klir, G.J. 1992. Fuzzy Measure Theory, Plenum Press, New York and london.

Wille, R. 1982. "Restructuring lattice theory: an approach based on hierarchies of concepts" In Rival I. (Ed.), Ordered Sets Reidel, Dordrecht-Boston, 445-470.

Yager, R.R. 1988. "On ordered weighted averaging aggregation operators in multi-criteria decision making." IEEE Transactions on Systems, Man and Cybernetics 18:183-190. 